Physics, edited by F. Seitz, D. Turnbull, and H. Ehrenreich (Academic, New York, 1969), Vol. 23.

${ }^{38}$ S. Andersson and J. B. Pendry, J. Phys. C 5, L41 (1972).

${ }^{39} \mathrm{~S}$. Y. Tong (private communication).

${ }^{40}$ S. Y. Tong, G. E. Laramore, and T. N. Rhodin (unpublished).

${ }^{41}$ See A. Goldsmith, T. E. Waterman, and H. J. Hirschhorn, Handbook of Thermophysical Properties of Solid Materials (MacMillan, New York, 1961), Vol. I, p. 459.
${ }^{42}$ C. B. Duke, G. E. Laramore, B. W. Holland, and A. M. Gibbons, Surf. Sci. 27, 523 (1971). See, in particular, Fig. 10. ${ }^{43}$ G. G. Laramore, J. É. Houston, and R. L. Park, J. Vac. Sci. Technol. 10, 196 (1973).

${ }^{44}$ J. E. Houston, G. E. Laramore, and R. L. Park, Surface Sci. 34, 477 (1973).

45 J. M. Baker and J. M. Blakely, Surface Sci. 32, 45 (1972).

${ }^{46}$ J. M. Burkstrand, Ph.D. thesis (University of Illinois, 1972) (unpublished); Phys. Rev. B 1, 3443 (1973).

\title{
Magnetic Breakdown and Phase-Coherent Galvanomagnetic Effects
}

\author{
C. E. T. Gonçalves da Silva* and L. M. Falicov \\ Department of Physics, ${ }^{+}$University of California, Berkeley, California 94720
}

(Received 22 December 1972)

\begin{abstract}
This paper presents a generalization of Pippard's network-model approach for the calculation of transport properties in metals in the presence of magnetic breakdown and partial phase coherence of the wave function. The generalization consists of introducing an ensemble of equivalent networks characterized by a well-defined fully coherent finite system of orbits. It allows for relatively easy computation of the transport effective path in the infinite-relaxation-time approximation. The idea is applied to two specific examples: the linear chain and the hexagonal network; the latter can be considered a good model for magnesium and can be compared with existing and future experiments.
\end{abstract}

\section{INTRODUCTION}

The aim of this paper is to present a model calculation of phase-coherence effects on the transport properties of systems in which magnetic breakdown is present. It is now over a decade since the existence of the phenomenon of magnetic breakdown (MB)-the interband tunneling of electrons in the presence of a strong magnetic field-has been recognized. ${ }^{1}$ In this decade the experimental studies ${ }^{2}$ have not only decisively confirmed the existence of the phenomenon, but also the quality of the data has by far surpassed the limits of the existing theoretical analyses. In the presence of $\mathrm{MB}$, oscillations in the magnetoresistance have been observed which are caused by quantum effects distinct from the density-ofstates oscillations that are observed in the de Haas-van Alphen or Shubnikov-de Haas effects. ${ }^{2,3}$ We can gain a qualitative understanding of these new effects if we consider the semiclassical picture of electrons moving along well-defined trajectories. ${ }^{4}$ When MB is operative, the electron may choose between different paths in moving from a given initial position to a given final position. This multiplicity of paths indicates that in a quantum treatment, observable interference effects should be present, as long as the electronic wave function, maintains its coherence along the various paths between given initial and final points. As Pippard ${ }^{5}$ has shown, the parameter that governs the coherence length is the density of dislocations in the crystal. In a crystal with a very high density of dislocations, the coherence length is negligible, no interference effects are present, and the transport properties, as calculated from the Boltzmann equation, reproduce the general features of the experimental result. ${ }^{6}$ As the density of dislocation is reduced, the coherence length increases and the first oscillations in the magnetoresistance, coming from paths that encircle the smallest areas, begin to appear. This regime has been successfuly treated by Falicov et al. ${ }^{7}$ If we try to improve on their approach there are two ways to go: we can either do quantum transport theory starting from a suitable approach, e.g. , Kubo's formula, or we can adopt a more modest, but ad hoc scheme for calculation of the conductivity based on semiclassical, e.g., Pippard's network, models. ${ }^{8}$ The advantages and disadvantages of both methods are quite clear. It we use the quantum transport approach, we have, to begin with, a well-defined theory for which, in principle, suitable approximation schemes could be devised. So far, however, nobody has succeeded in tackling the difficulties involved in such an approach. ${ }^{9,10}$ If, on the other hand, we start from the network model, we step into a vacuum, so to speak. The próblem is not completely well-defined and we must depend to some degree on intuition. Although the network model has been amply discussed in the literature and ad hoc justi- 


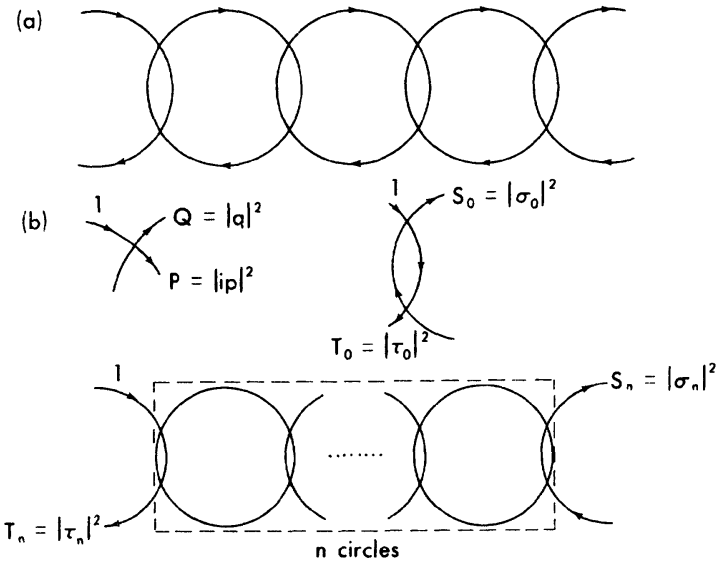

FIG. 1. (a) Linear chain; (b) amplitudes and probabilities for this network.

fications have been proposed, $4,8,9,11$ it remains essentially a semiclassical model in which quantummechanical properties are superimposed post hoc, à la Feynman, ${ }^{12}$ only along the azimuthal degree of freedom. Ultimately we must let the experimental evidence decide on the validity of our constructions.

In this paper is presented what seems to be the most natural generalization of the network model for finite coherence lengths. This is described in Sec. II.

In Sec. III we apply this generalization to the treatment of the linear chain. ${ }^{4}$ In Sec. IV we examine the hexagonal network using the same approach, because it would be interesting to compare our results with the experimental evidence, if and when it becomes available. As is well known, the topology of the hexagonal network is that of the most commonly used metal in these experiments, ${ }^{2,3}$ i.e., $\mathrm{Mg}$. In Sec. $\mathrm{V}$, we make some brief concluding remarks.

\section{PHASE COHERENCE AND EFFECTIVE PATH IN NET- WORK MODEL}

In the semiclassical approximation the orbit in $\vec{k}$ space of an electron moving in the presence of a magnetic field is given by the intersection of a plane perpendicular to the field and a surface of constant energy. ${ }^{1,13}$ The systems or orbits thus obtained (in the periodic-zone scheme) will be called a network. Figures 1 and 2 show the two types of networks examined in detail later on: the linear chain and the hexagonal network. Specific quantum effects can be considered by introducing the phase of the electronic wave functions in the WKB approximation. ${ }^{4}$ In this way we can easily reproduce Onsager's quantization condition, which restricts the possible orbits to those whose areas (in $\vec{k}$ space) satisfy

$$
a_{n}=2 \pi \frac{|e| H}{\hbar c}(n+\gamma),
$$

where $n$ is a non-negative integer and $\gamma$ is a small correction factor connected with the contribution of the radial motion. In the presence of $M B$ this simple picture is modified. The electron, instead of being totally reflected at the boundaries of the Brillouin zone, has a probability

$$
P=e^{-H_{0} / H}
$$

of tunneling into the next zone. In (2.2), $H$ is the applied magnetic field and $H_{0}$ is the magnetic breakdown field. ${ }^{2,14}$

This tunneling has drastic consequences for the topological properties of the orbits. We can see immediately that the influence of $\mathrm{MB}$ on the quantum aspects of the problem is striking, because the number of possible closed orbits now becomes enormous. The change in phase of the wave function of an electron traveling around a closed orbit is

$$
\Delta \varphi=\frac{\hbar c}{|e| H} a+\varphi_{0},
$$

where $\alpha$ is the area of the orbit in $\vec{k}$ space and $\varphi_{0}$

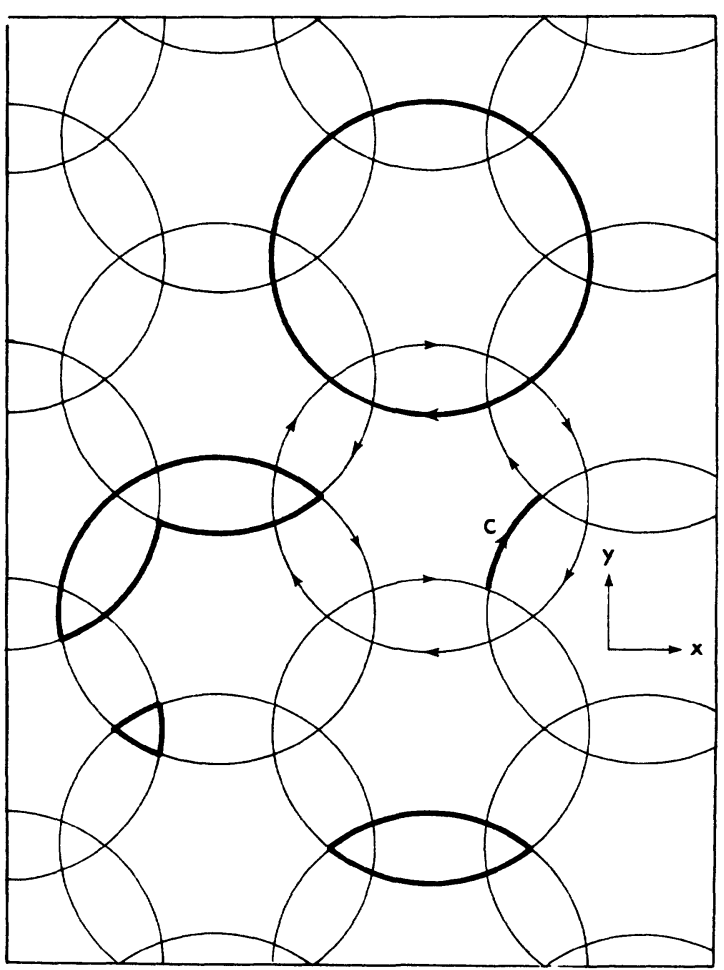

FIG. 2. Hexagonal network. We indicate the triangle, the lens, the two-lens, and the circle orbits. The branch $c$ is the standard branch. 
is a constant phase. (In Ref. 2 the reader will find a detailed prescription for the handling of the phases of the electronic wave function in the presence of MB. ) Naturally, only if the wave function remains coherent around the whole orbit is the quantization condition (2.1) applicable. Let us assume then that, in a given crystal sample, it is possible to define a coherence length $\xi$. It determines the region in space within which the electronic wave function maintains phase coherence. The quantity $\xi$ depends on the density of dislocations. At the moment there is no theory for it, although its existence can be safely assumed from the experimental evidence. Only those closed orbits which are entirely contained within a coherence length have to be treated quantum mechanically. Since the orbits in real space become smaller as the magnetic field increases, more and more such closed orbits become coherent. Now the consequences of this fact on the conductivity are investigated.

To compute the conductivity the effective-path approach due to Pippard is used. 5,7 This method is simply a way of solving the Boltzmann equation and hence is a classical (or semiclassical) method. It is extremely easy to apply in the infinite-relaxation-time limit. This means that scattering by phonons, impurities, etc., are neglected, i.e., mechanisms other than the magnetic breakdown process itself. The change in the electron distribution function caused by the presence of an external electric $\vec{E}$ is treated as the continuing creation of electrons and holes. These quasiparticles travel for a distance $\overrightarrow{\mathrm{L}}$ before their centers of mass come to a rest. From this point on they cease to contribute to the conductivity. The current is given by

$$
\overrightarrow{\mathrm{J}}=\frac{e^{2}}{4 \pi^{3} \hbar} \int \overrightarrow{\mathrm{L}}(\overrightarrow{\mathrm{E}} \cdot d \overrightarrow{\mathrm{S}}),
$$

where the integration is over the Fermi surface. The problem reduces to the computation of the effective path $\vec{L}$. Since we consider only the infiniterelaxation-time limit, $\overrightarrow{\mathrm{L}}$ is determined entirely by the probabilities of tunneling at each junction of the network. Of course, if we are interested in paths that lie wholly within a coherence length $\xi$, we must consider them as a single unit. The reason for this imposition lies in the hybrid nature of our calculation: the conductivity is calculated classically-hence we can only speak of probabilities-and the motion of the electrons has to be computed quantum mechanically-hence we need to sum amplitudes.

The advantage of the effective-path approach lies in the possibility of reducing its calculation to the solution of an algebraic equation. (The reader is again referred to Ref. 2, where this procedure is carefully described. ) However, this is only feasible if there is a periodic network. Although the semiclassical network is indeed periodic, as more and more orbits have to be considered fully coherent it is not obvious how periodicity can be restored. In what follows the scheme for doing this is described.

A given system of closed orbits is selected as a unit-denoted by $Z$. This unit is connected to the remainder of the network by a certain number of branches, along which an electron either enters into or emerges from it. To each branch $\beta$ of the network is associated an (as yet unknown) effective path $\overrightarrow{\mathrm{L}}_{\beta}$. If the probability that an electron coming into $Z$ from a branch $\beta$ and emerging from it in a branch $\beta^{\prime}$ is denoted by $P_{z}\left(\beta, \beta^{\prime}\right)$, the following equation for the effective path of the branch $\beta$ is found:

$$
\overrightarrow{\mathrm{L}}_{\beta}=\sum_{\beta^{\prime}} P_{Z}\left(\beta, \beta^{\prime}\right) \overrightarrow{\mathrm{L}}_{\beta^{\circ}},
$$

where the sum is over all branches $\beta^{\prime}$ that connect to $Z$. The probabilities $P_{Z}\left(\beta, \beta^{\prime}\right)$ can be computed straightforwardly, even if, for larger units, somewhat laboriously.

We have not yet said how the rest of the network is to be treated. We have made only the assumption that we can associate with each branch of the network an effective path. If the network is such that, by a judicious choice of the position of $Z$, we can take $\beta$ to be any branch in the network, we can understand (2.5) not as a single equation, but as a system of equations for all the effective paths in the network. In this case, periodicity is effectively restored and the various $\vec{L}_{\beta}$ are now related by simple geometrical transformations (translations and/or rotations). Equation (2.5) contains thus a single unknown $\overrightarrow{\mathrm{L}}$, the effective path of some branch which has been chosen as our standard. An equivalent, and perhaps more transparent, way of reintroducing periodicity is to consider an ensemble of networks in which each member is the complete network treated semiclassically, except for the unit $Z$ in one of its possible positions, which is treated quantum mechanically. We then ensemble-average Eq. (2.5) to obtain

$$
\left\langle\overrightarrow{\mathrm{L}}_{\beta}\right\rangle=\sum_{z} P_{z}\left(\beta, \beta^{\prime}\right)\left\langle\overrightarrow{\mathrm{L}}_{\beta^{\circ}}\right\rangle,
$$

where the angular bracket denotes the averaging. Again we are left with a single unknown effective path $\overrightarrow{\mathrm{L}}$.

This approach remains very simple if all branches of the network are equivalent. The procedure becomes more cumbersome when this is not the case.

This scheme is somewhat similar to the one employed by Falicov et al. ${ }^{7}$ in that a separation is made between regions in which the electrons move 


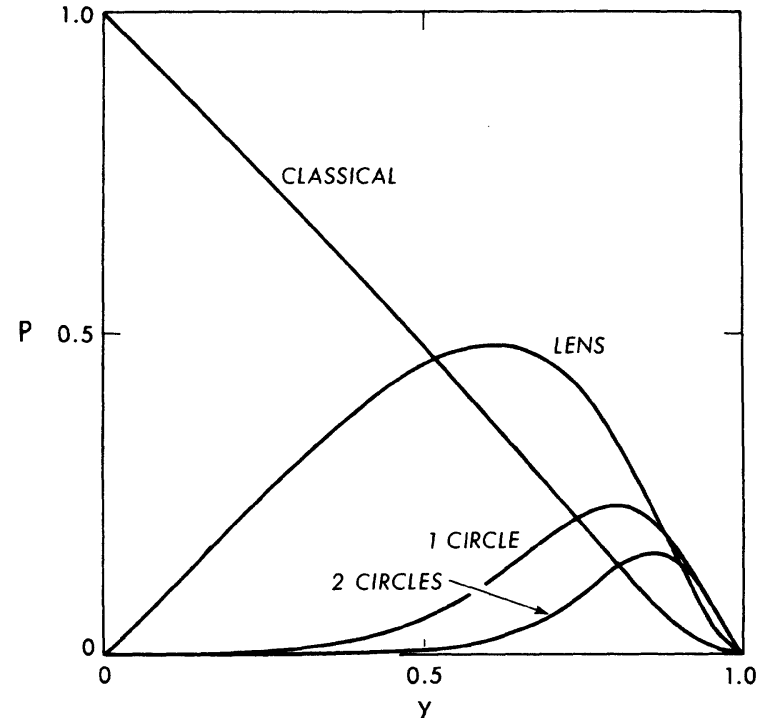

FIG. 3. Probabilities $P(Z)$ for various orbits of the linear chain as a function of $y=e^{-\left(H_{\mathrm{coh}} / H\right)^{2}}$.

quantum mechanically and regions in which they move classically. As a matter of fact, the ensemble-averaged network may be imagined as consisting of alternating quantum-mechanical and classical regions that preserve the periodicity, although, of course, no definite mental picture can be made of it.

\section{LINEAR CHAIN}

We apply the ideas developed in the previous section to the calculation of the magnetoresistance of the linear chain (Fig. 1). We make the assumption that the overlap of the circles is small, i.e., that only a small fraction of the Fermi sphere is contained in the second zone. With this assumption we can neglect the contribution of these portions of the Fermi surface to the conductivity, so that we have to consider only the topologically equivalent large arcs of the circle of the network in our calculation. With this approximation our approach becomes mathematically simple. The solution of (2.6) is now straightforward. The probabilities $P_{z}\left(\beta, \beta^{\prime}\right)$ are defined in Fig. [1(b)], where they are denoted by $S_{n}$ and $T_{n}$. The corresponding amplitudes $\sigma_{n}$ and $\tau_{n}$ can be easily computed. They are

$$
\begin{aligned}
& \sigma_{0}=q\left(1-\frac{p^{2} e^{2 i \eta}}{1-q^{2} e^{2 i \eta}}\right), \\
& \tau_{0}=-\frac{p^{2} e^{i \eta}}{1-q^{2} e^{2 i \eta}}
\end{aligned}
$$

and

$$
\sigma_{n+1}=\frac{\sigma_{0} \sigma_{n} e^{i x}}{1-\tau_{0} \tau_{n} e^{2 i x}}
$$

$$
\tau_{n+1}=\tau_{n}+\frac{\tau_{0} \sigma_{n}^{2} e^{2 i x}}{1-\tau_{0} \tau_{n} e^{2 i x}}
$$

where the amplitudes $p$ and $q$ are defined in Fig. 1 (b) and $n$ is a non-negative integer. The phase lengths of the small and the large arcs of circle are, respectively, $\eta$ and $\chi$. The transverse magnetoresistance is given by

$$
\rho=\frac{4}{\pi} \frac{H}{n|e| c r_{F}} L,
$$

where $n$ is the number of electrons per unit area, $r_{F}$ is the radius in real space of the orbit of an electron of the Fermi energy, and $L$ is the effective path.

Following our scheme, we choose a certain system of orbits as fully coherent (the lens, one circle, two circles, etc. ), and using Eqs. (3.1)-(3.4), we compute the probabilities $S_{n}$ and $T_{n}$. These probabilities determine the effective path and the magnetoresistance. However, in reality we are not interested in these "pure" results, but rather in an average which takes into account the existence of the coherence length $\xi$. Having computed the effective path for a given system of orbits $Z$, we assume now that the contribution of this unit $Z$ to the total conductivity can be described by a probability $P(Z)$. We thus set

$$
\overrightarrow{\mathrm{L}}_{\mathrm{av}}=\sum_{Z} P(Z)\langle\overrightarrow{\mathrm{L}}(Z)\rangle,
$$

where the sum is over all the possible distinct units $Z$ and $\langle\overrightarrow{\mathrm{L}}(Z)\rangle$ is the corresponding ensembleaveraged effective path. In order to justify such an expression we remark that, in a given crystal sample with a certain distribution of dislocations, we can use Pippard's criterion to decide whether

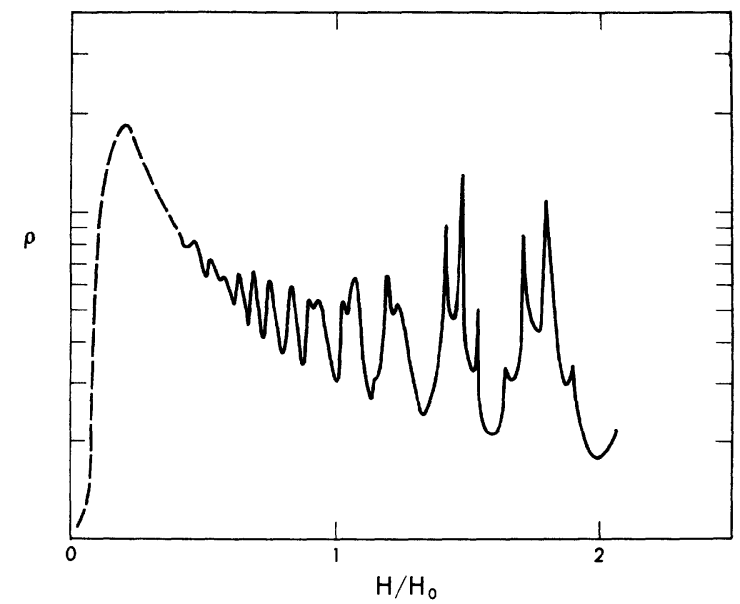

FIG. 4. Weighted average of the magnetoresistance (arbitrary scale) for the linear chain; $\eta=25\left(H_{0} / H\right)$ and $\chi=100\left(H_{0} / H\right)$. 


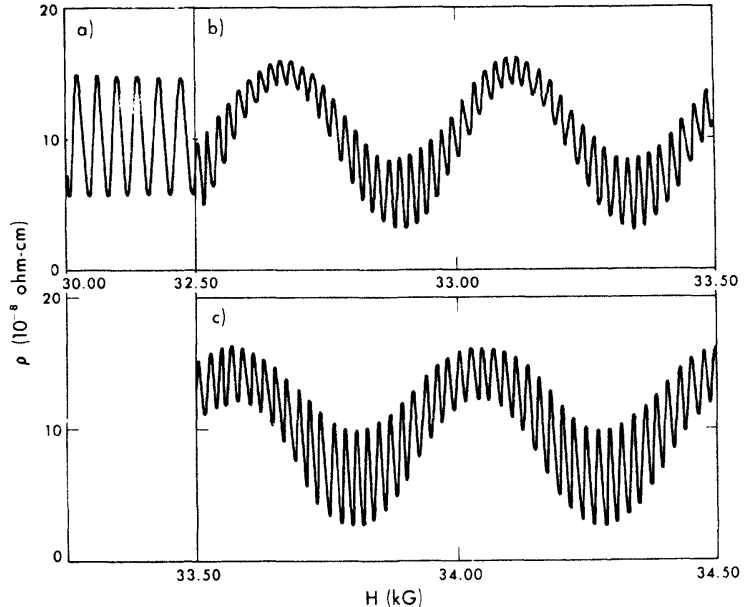

FIG. 5. Pure magnetoresistance for (a) the triangle, (b) the one-lens, and (c) the two-lens orbits in the hexagonal network.

or not the wave function maintains its coherence around a given closed orbit. This criterion says that the coherence is maintained if the orbit in real space encircles no dislocation. For a random distribution of dislocations, with a density $\rho$ per unit area, we use Poisson's formula to obtain the fraction of orbits with a given area $A$ (in real space) which are "coherent." This is given by

$$
f_{0}(A)=e^{-\rho A} \text {. }
$$

Of course, the fraction of orbits with area $A$ which are not coherent is

$$
f_{1}(A)=1-f_{0}(A) \text {. }
$$

For a given orbit of area $A(Z)$ to give a pure $Z$ signal to the conductivity it must be coherent. But this condition is only a necessary one; it is not sufficient. If the orbit is part of a larger orbit, which is itself fully coherent, then the observed signal is the one corresponding to the larger orbit. We can see that if $Z^{\prime}$ is the smallest unit within which $Z$ can be embedded, the probability $P(Z)$ is given by

$$
P(Z)=C f_{0}(A(Z)) f_{1}\left(A\left(Z^{\prime}\right)\right),
$$

where $C$ is a normalization constant. Because of the simple topology of the linear chain this expression can be easily evaluated. In Fig. 3 we have plotted the contributions of the various units as a function of

$$
y=e^{-\rho A_{l}} \equiv e^{-\left(H_{\operatorname{coh}} / H\right)^{2}},
$$

where $A_{l}$ is the real-space area of the lens. The coherence field $H_{\text {con }}$ is defined by the expression above.
TABLE I. Area of fundamental orbits in the hexagonal network.

\begin{tabular}{ll}
\hline & Area (a.u.) \\
\hline triangle & $6.49 \times 10^{-3}$ \\
lens & 0.1395 \\
circle & 1.660 \\
\hline
\end{tabular}

In Fig. 4 we have plotted the magnetoresistance as a function of $H / H_{0}$. We also indicate, in the low-field region, the semiclassical behavior of the magnetoresistance in the presence of magnetic breakdown. ${ }^{6}$ The choice of parameters was made purely having in view the graphical display of the results. In particular, the ratio of the phase lengths $\eta$ and $\chi$ is not consistent with the neglect of the contribution of the electrons in the second zone to the conductivity. For all coherent units, except the lens, there are values of the magnetic field for which

$$
S_{n}=1 \text { and } T_{n}=0 \quad(n>0) .
$$

For these values of $H$ we must introduce the finite relaxation time due to impurities, phonons, etc. , in order to avoid singularities in the magnetoresistance.

\section{HEXAGONAL NETWORK}

For the hexagonal network, Fig. 2, we can easily apply the ideas of Sec. II only if we neglect the contribution of the little triangular portions (third-zone electrons) to the conductivity. If we do so, all the remaining branches of the network

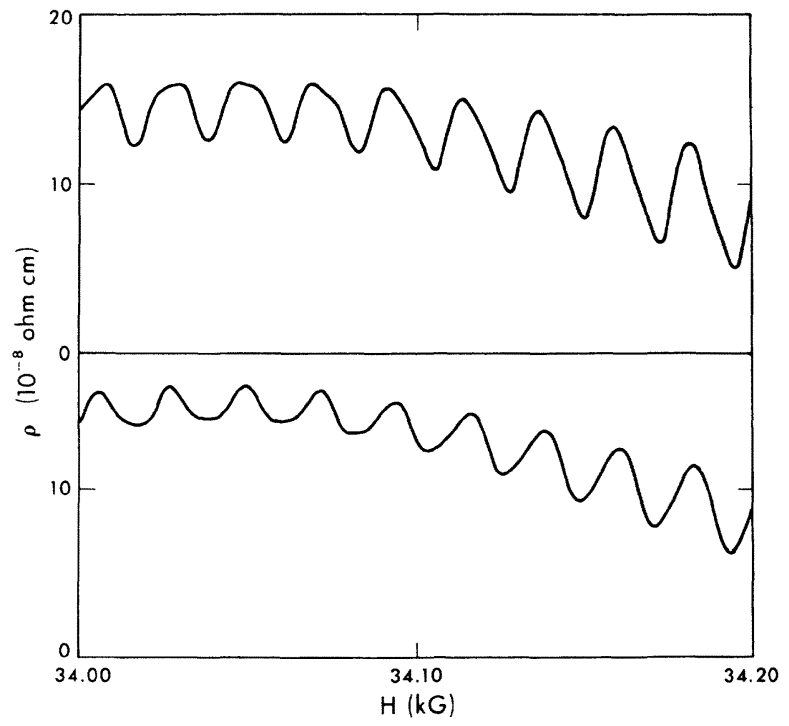

FIG. 6. Detail of the magnetoresistance oscillations for the one-lens (lower curve) and two-lens (upper curve) orbits in the hexagonal network. 


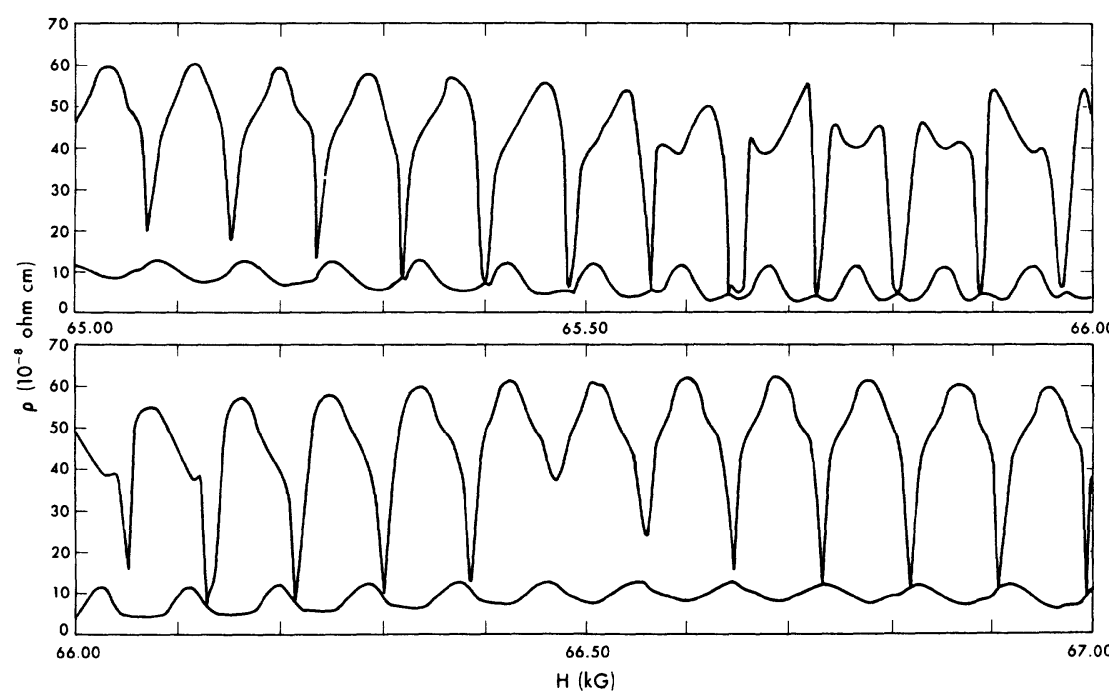

FIG. 7. Envelope of the circle oscillations in the hexagonal network.

are equivalent. The computation of the probabilities $P_{z}\left(\beta, \beta^{\prime}\right)$ is straightforward, although a simple recursion relation, as that found for the linear chain, does not exist. In computing the amplitudes we employ throughout the phase conventions of Ref. 2. Choosing as our standard the branch marked $c$ in Fig. 2, we find the contribution to the conductivity from a slab of thickness $\Delta k_{z}$ and with a density $n$ of electrons per unit area to be

$$
\Delta \sigma_{x x}=\frac{3}{2 \pi} \frac{n \Delta k_{e}|e| c}{H}\left[L_{x}+(\sqrt{3}) L_{y}\right]
$$

and

$\Delta \sigma_{y x}=\frac{3}{2 \pi} \frac{n \Delta k_{z}|e| c}{H}\left[L_{y}-(\sqrt{3}) L_{x}-2 \sqrt{3}\right]$.

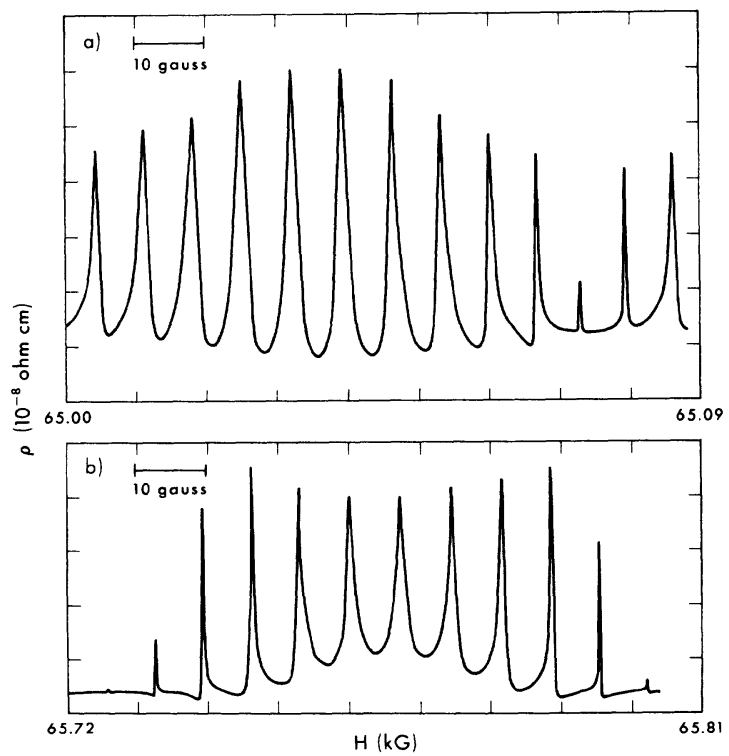

FIG. 8. Details of the circle oscillations in the hexagonal network.
The effective path for particles originating in the segment $c$ has been denoted by

$$
\overrightarrow{\mathrm{L}}=L_{x} \hat{x}+L_{y} \hat{y} \text {. }
$$

We ignore the contribution of the remaining portions of the Fermi surface to the conductivity. As Falicov et al. ${ }^{7}$ have shown, for the fields we are interested in $(H>10 \mathrm{kG})$ this contribution is completely negligible. We preserve, however, a very important feature characteristic of all hcp metals, e.g., Mg: the total compensation of electrons and holes at zero field.

The magnetic breakdown field $H_{0}$ is assumed finite and constant in the slab $\Delta k_{\mathrm{z}}$ and infinite everywhere else. For the slab we have set $^{7}$

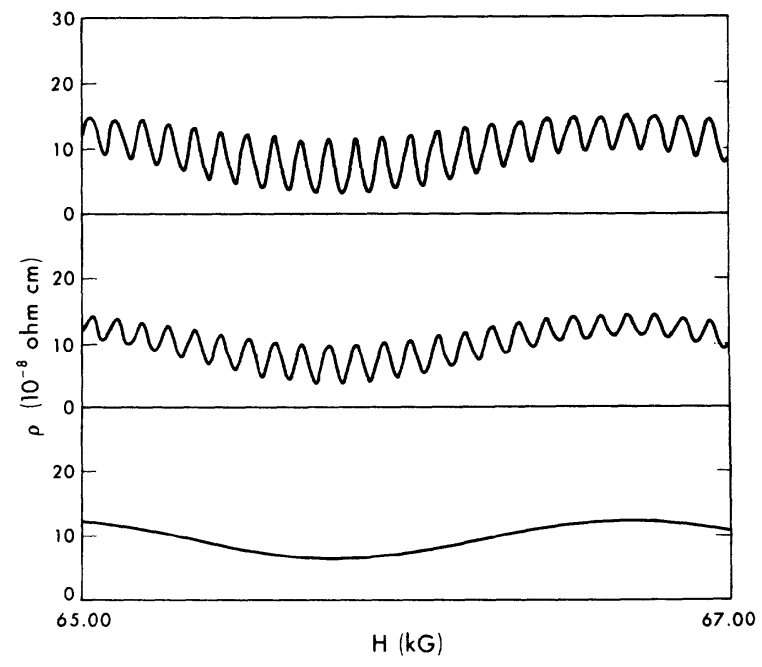

FIG. 9. Triangle (lower curve), one-lens (middle curve), and two-lens (upper curve) oscillations in the hexagonal network for the same range of fields as in Fig. 7. 


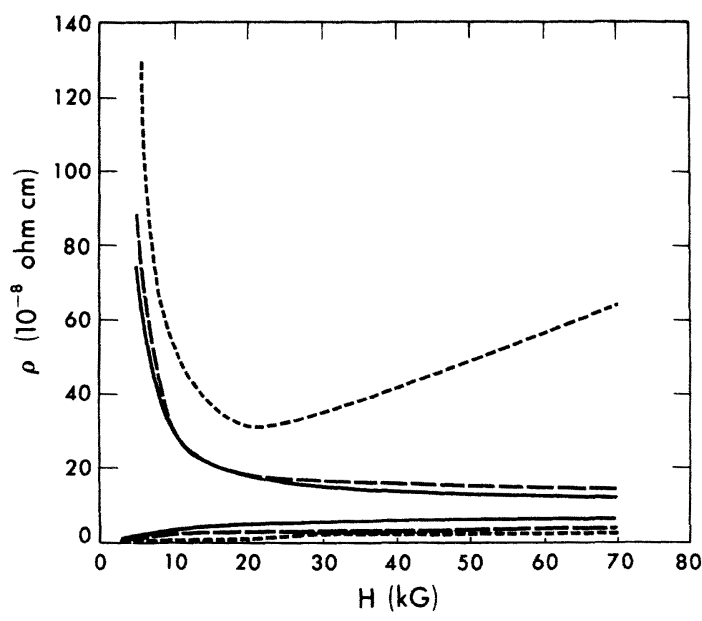

FIG. 10. Over-all envelope of the oscillations of the triangle (solid line), one-lens (dashed line), and circle (dotted line) orbits in the hexagonal network.

$$
H_{0}=5.3 \mathrm{kG} \text {. }
$$

We have chosen the parameter $n \Delta k_{g}$ in such a way as to obtain magnetoresistance values which are comparable to those of $\mathrm{Mg}$ :

$$
n \Delta k_{z}=3.79 \times 10^{21} \mathrm{~cm}^{-3} \text {. }
$$

Using the free-electron model, we can estimate that $\Delta k_{z} \cong 0.058 k_{F} \cong 0.04$ a.u. This is not an unreasonable value, since the interval $\Delta k_{\varepsilon}$ over which breakdown is probable in $\mathrm{Mg}$ is of the order of 0.1 a.u.

Finally, the remaining parameters which enter the calculation are the areas of the orbits in $\vec{k}$

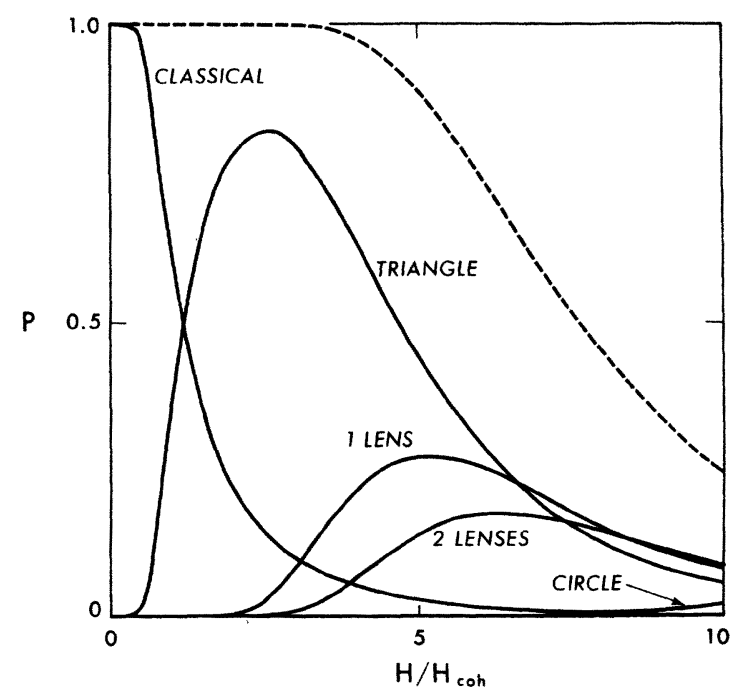

FIG. 11. Probabilities $P(Z)$ for various orbits in the hexagonal network. The dashed line is the sum of the contributions shown in the figure.

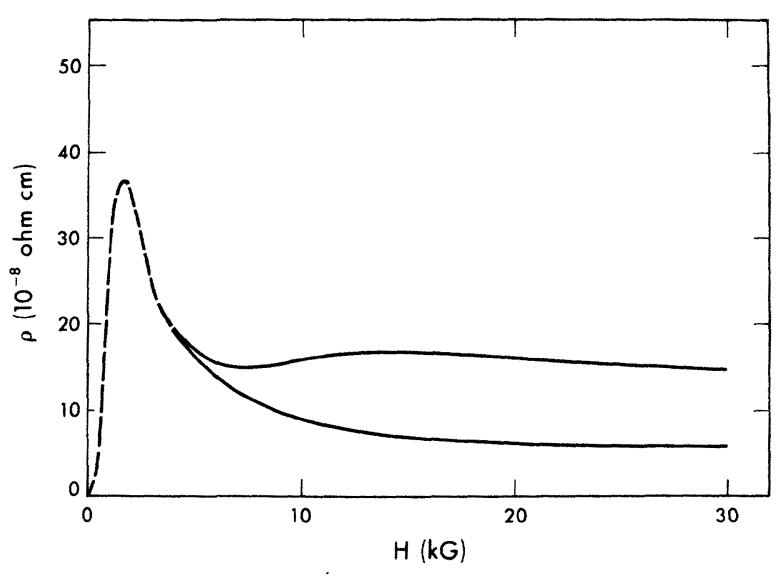

FIG. 12. Envelope of the averaged magnetoresistance of the hexagonal network.

space. There are just three basic orbits out of which all others can be built. In Table I we give: their areas. ${ }^{2}$

We adopt the convention that orbits described clockwise have positive areas (electron orbits) and orbits described anticlockwise have negative area (hole orbits). The phase change associated with an orbit of area $a$ is given by (2.3). We have set $\varphi_{0}=0$ throughout.

Figure 5 shows the results for the magnetoresistance taking the triangle, the lens, and the twolens orbits into account. Figure 5 (a) shows the triangle oscillations in the interval $30-32.50 \mathrm{kG}$. This curve is obtained if only the triangle orbits are coherent. In Fig. 5(b) we can see the consequences of the one-lens orbits being fully coherent. A fast oscillation with the lens period is superposed on the slow triangle oscillations. The amplitude of the fast oscillations is modulated by the triangle period. In Fig. 5 much the same is observed for the case in which the two-lens orbits are coherent. In this scale the only noticeable difference from Fig. 5(b) is in the amplitude of the fast oscillations, which are somewhat larger. There is also a line-shape difference, however, as Fig. 6 shows. The amplitude of the fast oscillations is still modulated by the triangle period. It is the general rule that whenever a higher frequency is introduced the amplitude of the oscillations with this higher frequency is modulated by the lower frequencies.

Figure 7 shows the envelope of the circle oscillations in the interval 65-67 kG. Figures 8(a) and 8(b) show the circle oscillations themselves in a greatly expanded scale. We have chosen two more or less typical results. Following the general rule above, the fast circle oscillation amplitude is modulated by the lens and also by the triangle oscillations. Figure 9 shows the triangle, one-lens, 


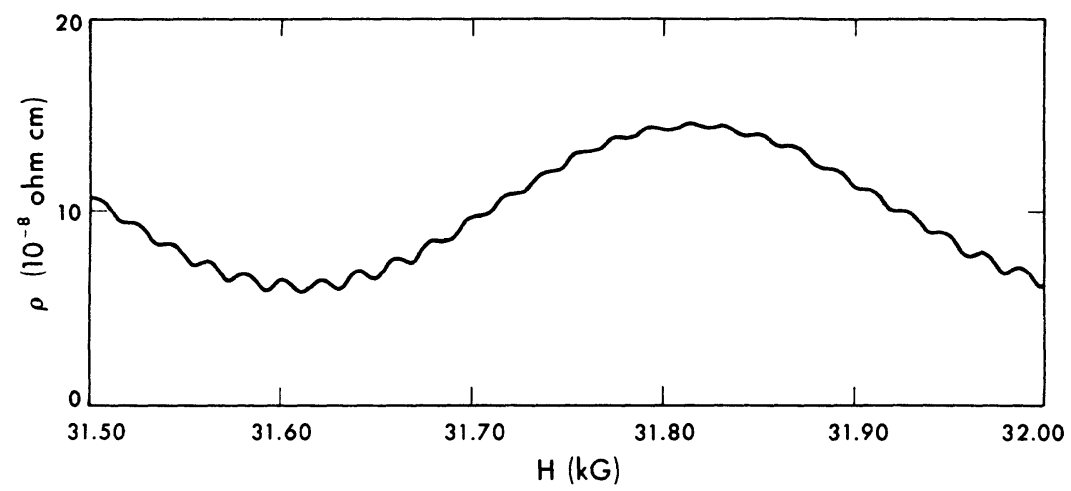

FIG. 13. Detail of the oscillations of the averaged magnetoresistance hexagonal network.

and two-lens results in the interval $65-67 \mathrm{kG}$. It is very easy to correlate the features of the circle oscillations with the phase of the triangle or lens oscillations. Finally, in Fig. 10 we show the overall envelope for the triangle, one-lens, and circle oscillations up to the fields of $70 \mathrm{kG}$.

Up to now we have presented only the pure oscillations. An averaging similar to the one described in Sec. III must be performed for the hexagonal network also. In Fig. 11 we show the probabilities $P(Z)$ for the orbits we consider here. The coherence field $H_{\text {coh }}$ is defined by an expression similar to $(3.10)$ in which the relevant area is that of the triangle. In Fig. 12 the envelope of the oscillations is plotted, and in Fig. 13 we show the oscillations in detail. The coherence field $H_{\text {coh }}$ was chosen so that for fields of the order of $30 \mathrm{kG}$, $H / H_{\text {coh }}$ is approximately 3 , and the classical curve plus the triangle, one-lens, and two-lens oscillations contribute 0.999 of the total probability. As we see from Fig. 11 , for $H>3 H_{\text {coh }}$ the contributions of orbits with areas larger than two lenses are indeed appreciable. Figure 12 should be compared with Fig. 26 of Ref. 2(a). The agreement is quite good, considering all the approximations involved in our calculation.

\section{CONCLUSION}

We have shown how the network model can be treated for arbitrary coherence length $\xi$. The introduction of an ensemble of equivalent networks allows for the restoration of the periodicity and hence for a straightforward computation of the transport properties, employing the effective-path approach. We applied this idea to the linear chain and the hexagonal network.
In the former case the simple topology permits the construction of a complete, closed-form solution. One curve for such a case is shown in Fig. 4 , where the highly nonlinear character of the oscillations is to be noted. The increasingly large number of coherent orbits as $H$ increases makes the resistance differ drastically from the simple sinusoidal behavior [see Ref. 2(a)], and at the same time eliminates unphysical zeros and infinities which appear when only one specific orbit is coherent.

The hexagonal case was treated approximately: besides the triangular orbits, we considered also the one-lens, two-lens, and circle orbits as fully coherent. Figures 5-10 once again clearly show the strong interdependence of the various oscillations and the interference effects between different coherent orbits. Comparison of these curves, and mostly of Fig. 12, with the available experimental data for $\mathrm{Mg}$ [see again Ref. 2(a)], shows good overall agreement. This gives a strong indication that our approach is correct. If better experimental curves, obtained on better crystals (with longer $\xi$ ), become available, as they most probably will, the procedures proposed and described in here can be straightforwardly used to obtain corresponding theoretical curves to be compared with the experiment.

\section{ACKNOWLEDGMENTS}

The authors would like to acknowledge the hospitality of Professor Højgaard Jensen and Dr. Henrik Smith at the Physics Laboratory I, H. C. $\emptyset$ rsted Institute, Copenhagen, where this work was initiated. One of us (C. E. T. G. daS. ) would like to thank Dr. H. Smith for several very interesting conversations on the subject of this paper.
*On leave of absence from Instituto de Fisica, UFRGS, Brasil, on a Conselho Nacional de Pesquisas Scholarship.

tWork supported in part by the National Science Foundation through Grant No. GH 34438.

${ }^{1}$ M. H. Cohen and L. M. Falicov, Phys. Rev. Lett. 7, 231
(1961).

${ }^{2}$ (a) For a review see R. W. Stark and L. M. Falicov, in Progress in Low Temperature Physics, edited by C. J. Gorter (NorthHolland, Amsterdam, 1967), Vol. V, p. 235; (b) A. B. Pippard, in The Physics of Metals I, edited by J. M. Ziman (Cambridge U.P., 
Cambridge, England, 1969).

${ }^{3}$ R. W. Stark and C. B. Friedberg, Phys. Rev. Lett. 26, 556 (1971).

${ }^{4}$ A. B. Pippard, Proc. R. Soc. A 270, 1 (1962).

${ }^{5}$ A. B. Pippard, Proc. R. Soc. A 287, 165 (1965).

${ }^{6}$ L. M. Falicov and P. R. Sievert, Phys. Rev. 138, A66 (1965)

${ }^{7}$ L. M. Falicov, A. B. Pippard, and P. R. Sievert, Phys. Rev. 151, 498 (1966).

${ }^{8}$ A. B. Pippard, Philos. Trans. R. Soc. Lond. A 256, 317 (1964).

${ }^{9}$ W. G. Chambers, Phys. Rev. 165, 799 (1968).
${ }^{10}$ A. O. E. Animalu, Ann. Phys. (N.Y.) 70, 150 (1972). We believe this paper to be in error since the expression used there for the current varies with the gauge chosen.

${ }^{11}$ H. W. Capel, Physica (Utr.) 42, 491 (1969); Physica (Utr.) 46, 169 (1970).

${ }^{12}$ R. P. Feynman and A. R. Hibbs, Quantum Mechanics and Path Integrals (McGraw-Hill, New York, 1965).

${ }^{13}$ J. M. Ziman, Principles of the Theory of Solids (Cambridge U. P., Cambridge, England, 1969), Chap. 9.

${ }^{14}$ E. I. Blount, Phys. Rev. 126, 1636 (1962).

PHYSICAL REVIEW B

VOLUME 8 , NUMBER 2

15 JULY 1973

\title{
Inhomogeneous Line Broadening in Donor Magneto-Optical Spectra*
}

\author{
David M. Larsen \\ Lincoln Laboratory, Massachusetts Institute of Technology, Lexington, Massachusetts 02173 \\ (Received 5 January 1973)
}

\begin{abstract}
We discuss inhomogeneous broadening of the spectral lines of shallow neutral donors in a magnetic field. The broadening arises from electric fields and field gradients produced by donor and acceptor ions in partially compensated semiconductors of high purity. On the assumption that the ions and neutral donors are randomly distributed in the semiconductor, line shapes are calculated and compared to observed line shapes in GaAs. Using the ion concentration as an adjustable parameter, we are able to account quantitatively for a number of observed effects on the random impurity model, but are led to suggest that some weak pairing between donor and acceptor ions is probably present.
\end{abstract}

\section{INTRODUCTION}

The study of the optical spectra of shallow donors and acceptors in semiconductors is by now an old subject. Nevertheless, the problem of understanding linewidths and line shapes in such spectra has scarcely been touched. With the arrival of relatively high intensity monochromatic sources of far-infrared radiation covering a discrete but broad spectral range (particularly the far-infrared gas lasers), the commercial availability of long-travel infrared interferometers and improvements in methods of purifying semiconductors (especially GaAs and $\mathrm{Ge}$ ) renewed study of spectral line shapes of shallow impurities in very pure semiconductors seems warranted.

Some high-resolution magnetospectroscopy of donor lines in very pure $n$-GaAs has already been reported ${ }^{1}$; the experimental findings have motivated the present work. Although our discussions will be aimed at understanding inhomogeneous line shapes in $n$-GaAs, the underlying principles should be applicable to any sufficiently pure partially compensated semiconductor with hydrogenic donors or acceptors.

Even the purest available $n$-type semiconductor materials are often found to contain significant concentrations of acceptor impurities. This means that for temperatures at which conduction-band electrons are "frozen out," such materials will consist of a host lattice containing a mixture of neutral donors, ionized donors, and ionized acceptors. Only the neutral donors can absorb photons in the far infrared so that the ionized impurities make their presence known in infrared-optical-absorption experiments exclusively by their perturbing effects on the neutrals. Most of this paper will be devoted to a discussion of how these perturbations affect the line shapes of optical transitions in the neutrals.

An electron bound to a donor will experience not only the Coulomb potential of the central donor ion, but also the sum of Coulomb potentials from all other charged impurity ions in the lattice. Assuming that they are distributed isotropically, ions which are relatively very far away from the neutral donor tend to contribute a part of the external potential which changes very little over the region of space occupied by the wave function of the donor electron. There are two main reasons for this. (1) Derivatives of the Coulomb potential decrease rapidly with increasing source-to-neutral-donor distance and (2) the potential gradients at the donor due to far-away charges tend to cancel out for an isotropic charge distribution. Because only spatial variation of the exterior potential ${ }^{2}$ over the donor volume can produce shifts in transition energies of the neutral donor, we can expect that only charges contained in a relatively small region around the donor will be effective in shifting the donor transition energy.

In Sec. I we write down the multipole expansion 